\title{
Salmonella: from basic science to clinical issues
}

\section{Josep Casadesús}

Departamento de Genética, Facultad de Biología, Universidad de Sevilla, Apartado 1095, E-41080 Sevilla, Spain = Tel.: +34954 557105 = Fax: +34954 557104 = casadesus@us.es

\section{Salmonella: From Basic Science To Clinical Issues}

\section{Villars-sur-Ollon, Switzerland, 3-7 October 2010}

Salmonella is a human pathogen that causes various types of infections, ranging from mild gastroenteritis to life-threatening typhoid fever. For decades, research on Salmonella has attracted not just clinical microbiologists and epidemiologists but also geneticists and molecular biologists who use Salmonella as a model organism. Hence, meetings dealing with Salmonella can be truly interdisciplinary. Such was the case in the ESCMID-FEMS Conference held in Villars-sur-Ollon (Switzerland) in October 2010. The meeting fostered interactive views of Salmonella biology and prompted the discussion of strategies to prevent and combat Salmonella infections.

Microbiologists from 16 countries (Brazil, Finland, France, Germany, India, Iran, Israel, Pakistan, Poland, Spain, Sudan, Sweden, Switzerland, Turkey, UK and the USA) gathered at the Swiss village of Villars-sur-Ollon, below the snowcapped Dents du Midi, to discuss Salmonella biology. The conference, entitled 'Salmonella: From Basic Science To Clinical Issues', was sponsored by the European Society of Clinical Microbiology and Infectious Diseases and the Federation of European Microbiological Societies, and was organized by Roland Koerner (Sunderland Royal Hospital, UK), Jean Claude Piffaretti (Université de Genève, Switzerland), Robert Read (University of Sheffield, UK), and Jordi Vila (Universitat de Barcelona, Spain). The program fully honored the title of the conference, and fostered a lively, interactive blend of molecular biology, epidemiology and clinical microbiology.

The opening lecture of the conference was delivered by Ferric Fang (University of Washington, WA, USA) under the title 'Salmonella: a window to biology'. Fang pointed out the relevant position of Salmonella as a model organism for both basic microbiology and clinical medicine, and reviewed the long list of biological concepts that have arisen from Salmonella research, from the 19th Century to present. Highlights in Fang's list were type III secretion, host specificity, macrophage responses to infection, silencing of horizontally acquired genes by nucleoid proteins, and signal integration by transcriptional regulatory cascades. Salmonella research continues, stimulated by the high impact of Salmonella infections on human health. Owing to the arsenal of analytical procedures now available for the study of Salmonella-host interactions, Fang concluded that Salmonella can be expected to continue teaching us new biological concepts in years to come.

\section{Mechanisms of pathogenesis}

The pathogenesis session was opened by Michael Hensel (Universität Osnabrück, Germany), who described the mechanisms employed by Salmonella to remodel the intracellular environment of mammalian cells. Effectors secreted by the type III secretion system (T3SS) encoded by Salmonella pathogenicity island 2 (SPI-2) modify the microtubule cytoskeleton, and induce changes in vesicular transport and reorganization of the endosomal system. The outcome of the process is the formation of a unique membrane-bound compartment, the Salmonella-containing vacuole, which has the features of an organelle whose structure and fate is controlled by the pathogen.

Andreas Bäumler (University of California, USA) described how Salmonella serovars that cause gastroenteritis use SPI-1- and SPI-2encoded TSS3 to elicit acute intestinal inflammation. This host response provides a new electron acceptor in the gut, enabling Salmonella to outcompete the natural microbiota of the intestinal lumen. This growth advantage facilitates Salmonella transmission to new hosts upon fecal shedding. The strategy of gastroenteritisassociated serovars is in stark contrast with that of Salmonella enterica serovar Typhi, the causative agent of typhoid fever. S. enterica serovar Typhi uses SPI-1 TSS3 to invade the intestinal epithelium and SPI-2 TSS3 to survive in
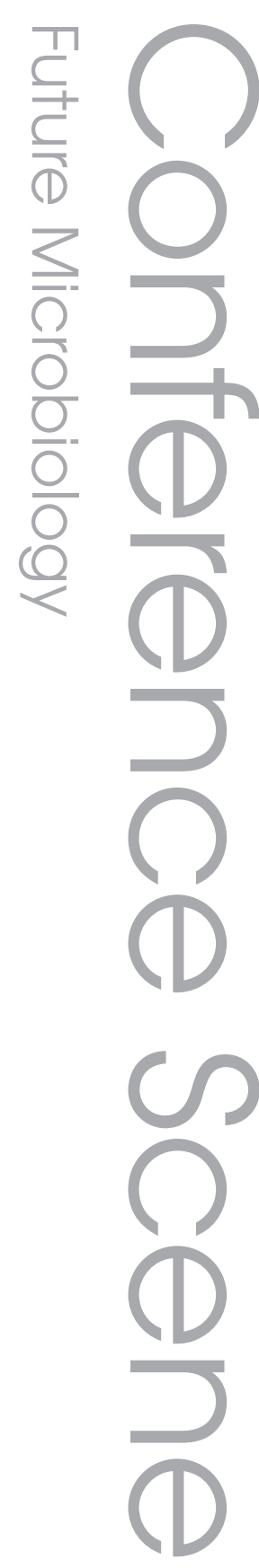
macrophages, and employs additional virulence factors to overcome the mucosal barrier and to colonize internal organs.

Pietro Mastroeni (University of Cambridge, UK) described Salmonella systemic infection as a heterogeneous and dispersive process that evolves in discrete phases, each characterized by a different level of bacterial division and death. Continuous spread to new infection foci (host phagocytes) is an essential trait in S. enterica virulence. Escape from infected phagocytes results in dispersive infections with low intracellular bacterial densities. Occurrence of low bacterial numbers per phagocyte may avoid competition for space and nutrients within the phagosome. Escape to new infection foci may also help the pathogen to stay one step ahead of the escalation of the local immune response.

\section{Antimicrobial resistance}

Laura Piddock (University of Birmingham, UK) reviewed the role of bacterial efflux pumps in multidrug resistance (MDR) and virulence. Pumps of the RND family confer innate MDR, thus explaining why Gramnegative bacteria are naturally resistant to many antibacterial agents effective against Grampositives. One such pump, AcrAB-TolC, is commonly overexpressed in MDR S. enterica serovar Typhi isolates of clinical and veterinary origin. Piddock also remarked that the role of AcrAB-TolC in Salmonella biology goes beyond MDR: lack of ArcAB-TolC causes virulence attenuation in the mouse model, and impairs Salmonella interactions with epithelial cells and macrophages.

Dan Andersson (University of Uppsala, Sweden) described antibiotic resistance as a two-step process. Mutation or horizontal gene acquisition usually confer antibiotic resistance at the expense of reducing bacterial fitness. In a second step, compensatory mechanisms ameliorate the fitness cost. One such mechanism is gene amplification, which has been shown to reduce the fitness cost of resistance to numerous antimicrobial agents. Amplification of either the target gene or unrelated genes can thus be viewed as a flexible, reversible strategy to restore fitness, obviously less risky than mutation.

Transferable drug resistance was addressed by Axel Cloeckaert (Institut National de la Recherche Agronomique, France). Since the 1990s, transferable drug resistances in Salmonella have been mainly due to the Salmonella genomic island 1 carrying various antibiotic resistance gene clusters, and to conjugative plasmids carrying genes that confer resistance to extended-spectrum cephalosporins. Salmonella genomic island 1, now found in many Salmonella serovars and in Proteus mirabilis, is an integrative mobilizable element. Conjugative plasmids carrying extended-spectrum cephalosporin resistance genes are widespread and have been found in Salmonella epidemic clones of animal and human origin. Although rare at the moment, further spread of such plasmids in MDR strains carrying Salmonella genomic island 1 is of concern.

Frank Aarestrup (National Food Institute, Denmark) analyzed the differences between global and local epidemiology of Salmonella antimicrobial resistance. In the last decade, antimicrobial resistance has increased globally. Although spread of a clone is not always easy to distinguish from the emergence of resistance in a previously susceptible clone, global spread of MDR clones has been observed in several cases (e.g., S. enterica serovar Typhimurium DT104). Reduced antimicrobial usage and limitation of transmission are major strategies to slow down the emergence of resistant clones. However, because global and local epidemiology show differences, these strategies should also be different depending on the serovar, the clone, the reservoir and the antimicrobial agent.

\section{Epidemiology \& clinical microbiology}

Rene Hendriksen (National Food Institute, Denmark) discussed the impact of international travel and trade on human salmonellosis. Travel to Asia and Africa presents the highest risk of infection. The reservoirs of Salmonella that cause human infections are often difficult to identify, because only a few serovars are host-adapted or host-restricted. Therefore, the WHO encourages countries to implement Salmonella surveillance programs. An interesting observation in recent WHO data is that $S$. enterica serovar Enteritidis and $S$. enterica serovar Typhimurium seem to be decreasing globally while $S$. enterica serovar Infantis is increasing.

Melita Gordon (University of Liverpool, UK) spoke on the emerging problem of invasive nontyphoid Salmonella disease in Africa among children and HIV-infected adults. Sequential epidemic outbreaks of MDR serovars led to the discovery of a novel pathovar, ST313, unique to and prevalent across sub-Saharan Africa. ST313 shows genome degradation and pseudogene formation similar to that seen in S. enterica serovar Typhi, suggesting novel and worrying microevolution towards a more invasive or 
host-restricted phenotype. Gordon also discussed the clinical pathogenesis of invasive nontyphoid Salmonella disease, and presented data demonstrating that invasive nontyphoid Salmonellae persist and replicate intracellularly in the bone marrow. Finally, Gordon highlighted the importance of viewing Salmonella disease in a global context, considering the huge economic costs of salmonellosis in Europe and the USA, the high burden of typhoid fever in Asia and the new problem of invasive nontyphoid Salmonella in sub-Saharan Africa.

John Threlfall (Health Protection Agency, UK) described the European situation concerning Salmonella antimicrobial resistance, and emphasized two aspects of particular relevance for public health. One is the increase in resistance to quinolones and cephalosporins, especially among isolates of serovar Enteriditis from eggs and egg products. Another problem is the emergence of new MDR clones of S. enterica serovar Typhimurium-like organisms ('monophasic' $S$. enterica serovar Typhimurium) associated with pigs and pig products. Two such strains have caused outbreaks and other infection incidents in at least ten European countries.

Nicholas Thomson (Sanger Institute, UK) reviewed the toolbox of analytical procedures for epidemiology, with emphasis on recent developments in high-throughput genome sequencing. The time of reference genomes is over, since nowadays it is possible to sequence multiple strains, pathotypes and isolates. Sequence analysis across species or genera can help to identify landmarks and risk factors of pathogen evolution. In turn, sequencing strains from outbreaks can link genotypic information to epidemiological data. Ongoing technological advances will make genome sequencing even faster and cheaper, thus providing a wealth of data for epidemiological and evolutionary studies.

\section{Other topics}

The conference was enriched with short talks on miscellaneous aspects of Salmonella biology. Owing to space limitation, only a few can be cited. Jessica Blair (University of Birmingham, UK) presented intriguing evidence that AcrB exports an unidentified, nonproteinaceous virulence effector. Elegant genetic analysis in the Salmonella histidine operon led Kelly Hughes (Université de Fribourg, Switzerland) to question the existence of neutral mutations in protein-coding genes. Christina Bronowski (University of Liverpool, UK) reported the characterization of the genome of $S$. enterica serovar Bovismorbificans, a zoonotic serovar that sporadically infects humans. Todd Callaway (US Department of Agriculture, USA) described the use of orange peel as a low-cost treatment to reduce the incidence of Salmonella and other foodborne pathogens in farm animals. Sohelia Moradi Bidhendi (Razi Vaccine \& Serum Research Institute, Iran) summarized recent studies on MDR Salmonella in Iran. Ohad GalMor (Sheba Medical Center, Israel) described the rapid emergence of MDR serovar Infantis in Israel. Altogether, short talks strengthened the interdisciplinary character of the conference, and further illustrated the excitement of Salmonella research depicted by Ferric Fang in the opening lecture.

\section{Financial \& competing interests disclosure}

The author has no relevant affiliations or financial involvement with any organization or entity with a financial interest in or financial conflict with the subject matter or materials discussed in the manuscript. This includes employment, consultancies, honoraria, stock ownership or options, expert testimony, grants or patents received or pending, or royalties.

No writing assistance was utilized in the production of this manuscript. 